\title{
A CFD Lagrangian particle model to analyze the dust dispersion problem in quarries blasts
}

\author{
J. T. Alvarez, I. D. Alvarez, S. T. Lougedo \& B. G. Hevia \\ GIMOC, Mining Engineering and Civil Works Research Group, \\ Oviedo School of Mines, University of Oviedo, Spain
}

\begin{abstract}
In the framework of the Research Project CTM2005-00187/TECNO, "Prediction models and prevention systems in the particle atmospheric contamination in an industrial environment" of the Spanish National R+D Plan of the Ministry of Education and Science, 2004-2007 period, a CFD model to simulate the dispersion of the dust generated in blasts located in limestone quarries has been developed. This is a complex phenomenon that is being studied through the use of several digital video recordings of blasts and the dust concentration field data measured by light scattering instruments, as well as the subsequent simulation of the dispersion of the dust clouds using Multiphase Computational Fluid Dynamics. After several tests with multiphase methods, both Eulerian and Lagrangian, finally the latter was used due to its ease in implementing calculations of discrete phases composed by multisized particles with affordable memory requirements.
\end{abstract}

Keywords: Bench Blasting, dust dispersion modelling, CFD, Discrete Lagrangian methods.

\section{Introduction}

This paper explains the simulations done to model the dust dispersion generated in a production blast located in a medium sized limestone quarry, ranging around $1 \mathrm{Mt} /$ year, exploited through drill and blast method. Limestone is a key natural resource, base of multiple materials used in Civil Works and main raw material of the cement.

In order to achieve this production level there are needed at least two blasts per week, blasts that generate several possible environmental risks as the aerial 
wave, the ground vibrations and the dust thrown to the atmosphere and dispersed by the wind.

Our research group is developing a project named CTM2005-00187/TECNO, "Prediction models and prevention systems in the particle atmospheric contamination in an industrial environment" granted by funds of the Spanish National R+D Plan of the Ministry of Education and Science, 2004-2007 period. Within the research objectives appears one relating to the determination of the amount of dust produced in a blast and its immersion in the atmosphere surrounding the quarry area by means of two main tools: measurement campaigns of the dust concentration using "Light scattering" dust sensors and computerized simulations through commercial CFD software. These are done through the combined use of Solidworks to generate the 3D models, ICEM CFD to adequately mesh the domain and Ansys CFX 10.0 in case of the calculation and analysis of the results.

There are several numerical methods that can be used to study the particulated material dispersion. One good summary of them was done by Reed [1] and the authors have already used classical dispersion models with acceptable results [2], but much more sophisticated tools are needed in order to study in more detail the dispersion effects and the possible mitigation methods. Among the possible methods it was decided to use the "Particle Tracking" one, a Lagrangian method implemented in CFX 10.0 with undoubted advantages in case of simulations of multisized dispersed phases.

\section{Blast characteristics}

The start conditions basic or determinate a dust dispersion problem are two: the emission conditions, which in this case depend on the parameters that define the blast, and the atmospheric conditions.
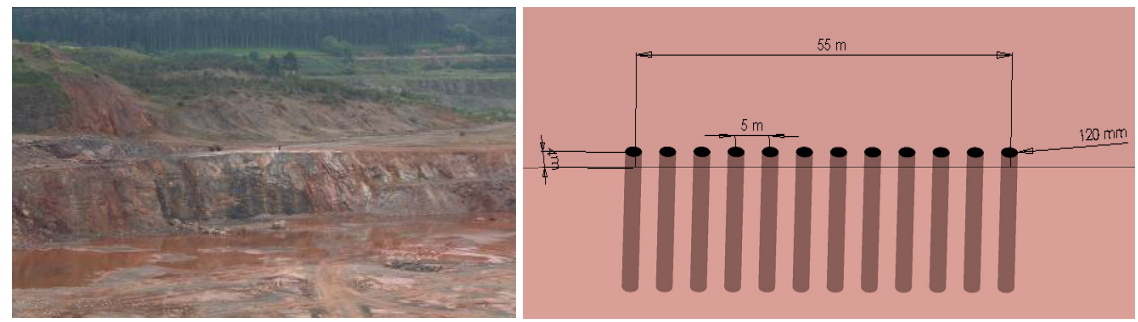

Figure 1: Quarry bench and blast design.

Table 1 shown below summarizes the main blast parameters. This is a classical bench blast, see figure 1, with only one row of shots that are loaded with two explosive types: one high power explosive placed at the bottom based on nitro-glycerine and another one of medium power based on ammonium nitrate that creates the so called column load. The upper part of the shot is filled with a compacting material, usually the product resulting of the drill, which is called clay stopper. 
Regarding the atmospheric conditions during the blast there were registered $24^{\circ} \mathrm{C}$ of ambient temperature, 1015 milibars of ambient pressure, sun and null rainfall. Wind velocity was measured using a Met-One wind sensors and was appreciably constant at $2.5 \mathrm{~m} / \mathrm{s}$. The wind bearing was at right angle to the bench face, which was completely dry. Relative humidity was measured at $55 \%$. These meteorological parameters will define the wind profile used in the simulation.

Table 1: $\quad$ Blast characteristics.

\begin{tabular}{|l|l|l|l|}
\hline Bench height & $18 \mathrm{~m}$ & Bottom charge depth & $5 \mathrm{~m}$ \\
\hline Burden & $4.5 \mathrm{~m}$ & Column charge depth & $9 \mathrm{~m}$ \\
\hline Spacing & $5 \mathrm{~m}$ & Depth detonator & EZDET \\
\hline Drill diameter & $120 \mathrm{~mm}$ & Head detonator & $\begin{array}{l}\text { MS-16 } \\
\text { ms })\end{array}$ \\
\hline Shot slope & $15^{\circ}$ & Initiation type & Electric \\
\hline Stemming & $2,83 \mathrm{~m}$ & Shot number & 12 \\
\hline Drill depth & $18 \mathrm{~m}$ & Total explosives load & $1586 \mathrm{~kg}$ \\
\hline Bottom charge & $50 \mathrm{~kg}$ & Detonating cord & $19 \mathrm{~m} / \mathrm{shot}$ \\
\hline Column charge & $82 \mathrm{~kg}$ & & \\
\hline
\end{tabular}

Another important parameter that has to be defined is the particle size distribution of the material that forms the dust cloud. As is shown by Almeida [3] et al and Jones et al [4] the particulated material thrown to the air by a blast has two main sources. First, rock pulverized by the several phenomena that take place in the blast (shock wave, high pressure gases or dynamic breaking mechanisms, etc.) and second the dusty products of the explosive chemical reaction. Both Almeida and Jones estimate the size distribution of the dust clouds in ranges from submicron sizes up to 50 microns. Particles over this size are also produced but have not been considered in the simulation as are quickly settled into ground by its own weight.

The several blasts studied were registered in digital video. There can be clearly noticed in those recordings the dust cloud generation and the subsequent movement and dispersion by the wind. Figure 2 shows a couple of still images taken from the tapes.

In addition to the digital video there was also used continuous measurement instrument to record the dust concentration values. The technology "light scattering" was employed, two equipment named E-Sampler manufactured by Met One Instruments Inc., both equipped with electronics capable of transferring the concentration meteorological data to a computer.

These dust sensors were located at several distances form the bench one at $120 \mathrm{~m}$ from the blast and the other one at $200 \mathrm{~m}$, distances that were confirmed as enough in order to Project the equipment form the flyrocks. There were obtained concentration peaks between 500 and $900 \mu \mathrm{g} / \mathrm{m}^{3}$ in case of the 
$120 \mathrm{~m}$ sensor and much lower values, between 150 and $400 \mu \mathrm{g} / \mathrm{m}^{3}$ in case of the equipment installed at $200 \mathrm{~m}$. Therefore it can be inferred that the dust cloud is starting its dispersion as it moves away from its source.
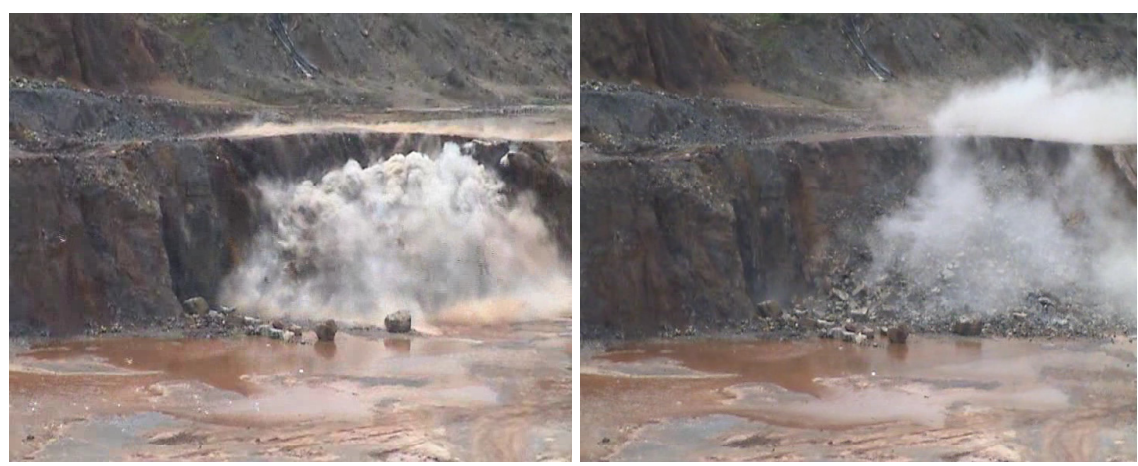

Figure 2: $\quad$ Blast and dust cloud.

\section{CFD simulation}

\subsection{Approach}

The numerical modelling of the dispersion of contaminant species in the atmosphere is with no doubt reaching its climax and several different techniques are available in the software market.

The particle tracking method is a multiphase modelling tool implemented in Ansys CFX 10.0, where a dispersed phase, the particles in this case, follow in a Lagrangian way the pressure and velocity field calculated in the continuum phase, which is the air. The particle trajectories are not evaluated for each and every one of them, but only for a limited number that will be representing the millions that compose the dust cloud. Each representative particle path will be assigned a certain amount of mass, and the movement of this mass through the continuum domain will be studied in a time-dependent way, as the emission will be considered not constant. Ansys CFX can represent dispersed phases as solid particles, liquid particles or even bubbles. In this case it will be used a dispersed phase made from 7 families of particles sizes, with mass distributions obtained from Almeida and Jones.

The method is used following the classical procedure used in the CFD simulations: geometry definition, meshing of the domain, definition of the problem physics, solution and finally post processing of the results.

\subsection{Geometry and meshing}

Starting from the information taken form the field and the surveying of the area it was developed a 3D model of the bench and its surrounding air using Solidworks. This parametric software woks through the use of Boolean operations among geometries in a similar way as a sculptor creates its works. 
Dimensions are approximately 400x500x250 m. In order to simulate the blasted material it will be created a semicone in the bench area with a $56^{\circ}$ slope, as it can be seen below in figure 3 .

In the same figure is shown the final 3D model created, with different colours for each geometry feature. Please notice the semicircle shape present over the bench, which will be defined, as the other surrounding volumes, as air but will be used only as a meshing tool to define different density in the mesh structure.

Once the geometry is created it is exported to the meshing tool, ICEM CFD. This software allows the creation of structured or unstructured grids composed by tetrahedrons, prisms or hexahedrons or a mixture of any of these elements. It will be defined a much more fine grid in the areas where the dust is present, not only in the blast area but also in the areas occupied by the dust cloud, as there is the place where it can occur more steep gradients in both the continuum and disperse phase defining variables.

Another parameter that has to be taken into account is the quality of the mesh, quality understood as the proximity of the tetrahedral elements that compound the mesh to the perfect tetrahedron. The use of perfect tetrahedral allows an homogeneous placing of the calculation nodes over the domains, which benefits the easiness of convergence of the CFD calculation and frequently can be a key issue that can define even the existence of a solution [6]. Figure 4 shows the final mesh used, after two previous attempts that were rejected due to the calculation differences shown.

The final mesh includes a global value of 706.324 elements with a final RMS quality of 0.38 . A much finer grid will make almost unfeasible the calculation in the computer used, Pentium $42.4 \mathrm{GHz}$ and 1GB RAM, making essential the use of calculation clusters in a multiprocessing scheme. Calculation duration ranged from 6 to 8 hours depending on the simulation time.
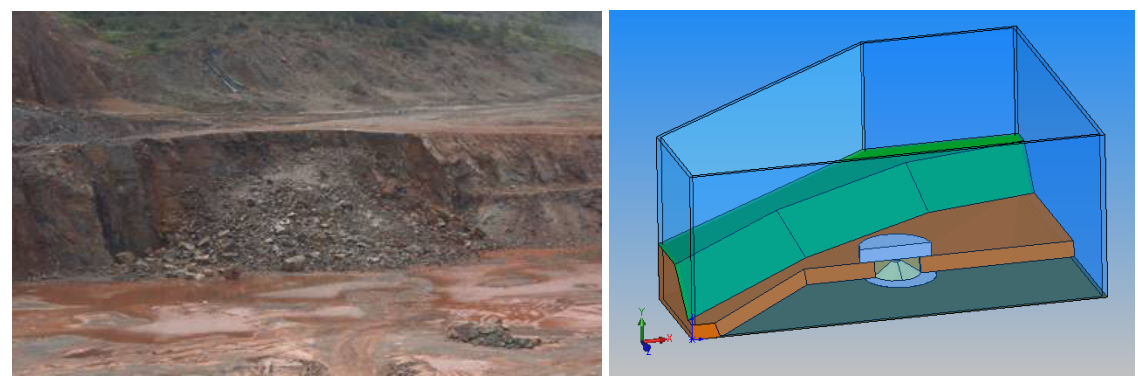

Figure 3: Bench after the blast and 3D model.

\subsection{Problem physics and resolution}

Once the domain is created and is adequately meshed the next step is to define the boundary conditions. These conditions are necessary to define how the different geometrical areas that limit the simulation domain are being affected by the variables affecting both the continuum and dispersed phase. Each surface has to be defined as an "Inlet", "Opening", "Outlet" or "Wall". 


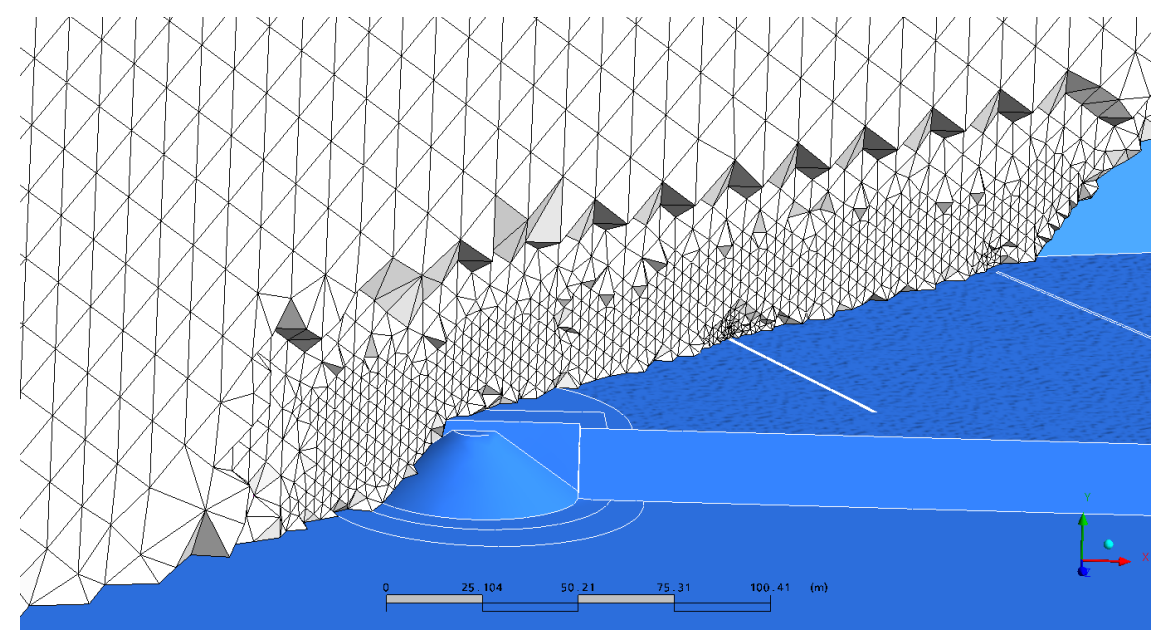

Figure 4: $\quad$ Model meshing.

There will be considered two Inlet conditions, the surfaces closer to the spectator in the figure 4, where the air enters in a subsonic regime. The blowing air will be defined as a velocity profile following a classical logarithmic equation, as can be seen in documents from the U.S. Environmental Pollution Agency [7] as well as adequately oriented following the wind bearing measured in the field.

The remaining vertical surfaces and the ceiling will be defined as "Openings", areas where there will be free flow both incoming or out coming. The remaining surfaces will be defined as "Walls" with a roughness factor of $0.5 \mathrm{~cm}$. The software automatically applies wall functions to define adequately the air flow near these surfaces.

As has been pointed out the simulation will be done using a dispersed phase, the dust cloud, moving through a continuum, the air. A key parameter that has to be defined is the turbulence model used to calculate the continuum variables field. Medium complexity turbulence models were selected in order to obtain affordable resolution times in single processor machines (Temmerman et al [8] Silvester et al [9]). Similar studies done by the authors in similar applications (see $[10,11]$ ) guided to the final selection of a roughness k-epsilon model using a logarithmic wind profile.

Simulations were methodically repeated using several dust mass flow values until the concentration curves calculated were almost equal to the measured ones. These curves were compared simultaneously at both measurement points located at 120 and $200 \mathrm{~m}$ from the blast.

The dust injection is achieved through the combination of several surface sources both planar and spherical in the blast surrounding area. The dust is injected in a pulsed shape with a duration ranging 25 to $35 \mathrm{~s}$. 


\subsection{Results and interpretation}

The analysis of the results of a CFD simulation is generally speaking easy and intuitive when using the modern post processing tools. The valuable time spent on the 3D-modelling, meshing and the establishing of the physics of the simulation is worthwhile once it is realized the wide possibilities of visualization and interpretation of calculation results.

Among the possible studies that can be done there can be highlighted the velocity vectors fields in planes parallel to the benches, the contours of the concentration variables in each time that will show the position of the dust cloud, contours of the maximum concentration values independently of time, etc. Due to space limitations, in this paper there will be shown just the concentration isosurfaces in several cases of simulation times and concentrations, isosurfaces that are considered representative of both the simulation results and the potentiality of the methodology used.

The CFX-Solver stores in the output files the values of the variables that define the behaviour of the continuum phase in each and every one of the calculated points defined by the volume finite technique. It also assigns to each control node of the mesh a value of the volume fraction of the dispersed phase in the continuum. This value will serve as base to develop that calculation of the dust concentration expressed, as is usual in these environmental studies in ranges of $\mu \mathrm{g} / \mathrm{m}^{3}$.

One of the most useful features of the postprocessor is the isosurface generation of a certain concentration value. This is, there can be shown the surface that fit all the points in the simulation domain where the concentration value is equal to a selected value. This will make appear in the display the shape and position of the dust cloud.

Figure 5 shows several isosurfaces of concentrations ranging $100 \mu \mathrm{g} / \mathrm{m}^{3}$ to $750 \mu \mathrm{g} / \mathrm{m}^{3}$. Two columns of figures appear, the left one representing simulation time at $55 \mathrm{~s}$ and the right one at $75 \mathrm{~s}$ with concentration values increasing towards the bottom of the figure.

Comparing the two figures appearing in the row signed as $0.1 \mathrm{mg} / \mathrm{m}^{3}$ we can observe how the isosurface of $100 \mu \mathrm{g} / \mathrm{m}^{3}$ has been displaced towards the right side of the figure as the dust cloud is moved by the air.

The second row of figures, clouds with concentration levels at $200 \mu \mathrm{g} / \mathrm{m}^{3}$ show a similar behaviour, although the size of the isosurface is clearly smaller, as it was expected, as it has to be included within the surface relative to 100 $\mu \mathrm{g} / \mathrm{m}^{3}$.

The third row of figures, $500 \mu \mathrm{g} / \mathrm{m}^{3}$, shows a much more shrink cloud and the dust concentrated in the low part of the bench. It is also useful to point out how the dust clouds are smaller as the time gets higher, which is showing the effects of the dispersion of the dust.

The fourth and last row shows high concentration values, $750 \mu \mathrm{g} / \mathrm{m}^{3}$, that are again only appearing in the figures of the left side, not being almost represented in the right ones. This is, as time evolves, higher concentrations disappear from the domain. 
16 Computational Methods in Multiphase Flow IV

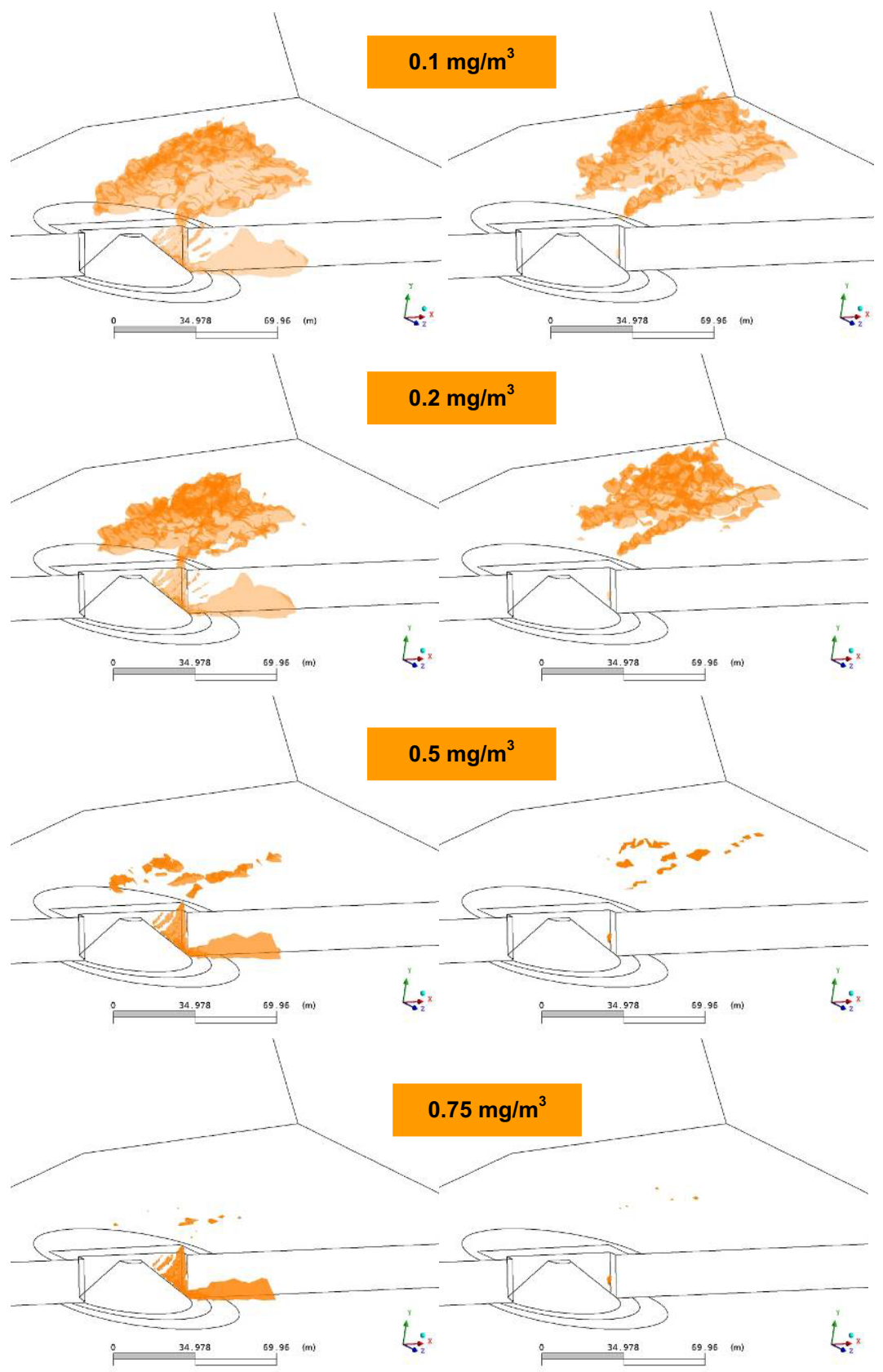

Figure 5: Dust cloud evolution at Time $=55 \mathrm{~s}$ (left column) and $75 \mathrm{~s}$ (right one). 


\section{Conclusions}

The dispersion of the particles generated in blasts located in quarries can be simulated using CFD tools as Ansys CFX 10.0. The use of digital video, detailed topography and continuous measurement of dust concentration allow the tuning and verification of the developed models.

The use of transitory particle tracking models allows the detail study of the dust cloud movement and will allow future studies conducted to mitigate the possible environmental impact generated.

\section{Acknowledgements}

We want to acknowledge the help and advices from the Ansys CFX Technical Support Team in the development of these studies.

\section{References}

[1] Reed, W.R. Significant Dust Dispersion Models for Mining Operations. Information Circular 9478. National Institute for Occupational Safety and Health (NIOSH). September 2005

[2] J. Toraño, R. Rodriguez, I. Diego and A. Pelegry, "Contamination by particulated material in blasts: analysis, application and adaptation of the existent calculation formulas and software". Environmental Health Risk III, pp. 209-219, (2004).

[3] Almeida, S.M. Eston, S.M. and De Assunçao, J.V. "Characterization of Suspended Particulate Material in Mining Areas in Sao Paulo, Brazil." I.T. International Journal of surface Mining, Reclamation and Environment 2002, Vol. 16, no. 3, pp. 171-179

[4] Jones, T., Morgan, A. and Richards, R. "Primary blasting in a limestone quarry: physicochemical characterization of the dust clouds". Mineralogical Magazine, April 2003, Vol 67(2), pp. 153-162

[5] ANSYS CFX-Solver, Release 10.0: Theory; Particle Transport Theory: Lagrangian Tracking Implementation; page 173.

[6] ANSYS CFX-Solver, Release 10.0: ANSYS CFX-Solver, Release 10.0: Modelling Page 327.

[7] Environmental Pollution Agency. AP-42, 13.2.5.1, Miscellaneous Sources. Pp2. 1998.

[8] Temmerman L., Wang C. and Leschziner M.A. (2004) A Comparative Study Of Separation From A Three-Dimensional Hill Using Large Eddy Simulation And Second-Moment-Closure Rans Modelling. European Congress on Computational Methods in Applied Sciences and Engineering. ECCOMAS

[9] S.A. Silvester, I.S. Lowndes and S.W. Kingman, "The ventilation of an underground crushing plant", Mining Technology (Trans. Inst. Min. Metall. A), Vol. 113, pp. 201-214 (2004) 
18 Computational Methods in Multiphase Flow IV

[10] Surface velocity contour analysis in the airborne dust generation due to open storage piles. Toraño J., Rodríguez R. and Diego I. European Conference on Computational Fluid Dynamics. ECCOMAS CFD 2006, Delft The Netherland, 2006

[11] Toraño J. et al., Influence of the pile shape on wind erosion CFD Emission Simulation, Appl. Math. Modell. (2006), doi:10.1016/j.apm.2006.10.012 